\title{
Efficacy and Safety of Autologous Serum Eye Drops in Treatment of Severe Dry Eye
}

\author{
Parul Singh M. S.*, P. D. Sharma M. S.*, Achyut N Pandey M. S., \\ Ameeta Koul M. S. \\ * Deptt of Ophthalmology. V.C.S.G.G.M.S.\&R.I., Srinagar Garhwal, Uttarakhand, India.
}

\begin{abstract}
:
Aim: To evaluate the efficacy and safety of autologous serum eye drops in treatment of severe dry eye.

Material and methods: Twenty six eyes of thirteen severe dry eye patients were enrolled in this study. All patients were refractory to treatment with conventional artificial tears. Autologous serum eye drops were applied eight times a day per eye. Patients were evaluated subjectively and objectively with Schirmer's test, TBUT, Fluorescein stain at start of treatment, at two weeks, and six weeks after start of treatment.

Results: Clinically and statistically significant improvement was seen both objectively and subjectively in patients of severe dry eye treated with autologous serum.

Conclusion: Autologous serum eyedrops were found effective and safe in treatment of severe dry eye disease, as evidenced by improvement in subjective assessment of symptoms, Schirmer's score, tear film break-up time and ocular surface staining.
\end{abstract}

Keywords: Dry eye, autologous serum, Schirmer's test.

\section{Introduction:}

Tear film plays an important part in the maintenance of intact ocular surface with their anti-microbial, nourishing, mechanical and optical properties. Components of tears include growth factors, fibronectin and vitamins to support proliferation, migration and differentiation of the corneal and conjunctival epithelium. Depletion in quantity or quality of tears can lead to various changes like squamous metaplasia, epithelial defects and infections of corneal epithelium. Artificial tears used in treatment of dry eye provide lubrication to the ocular surface, but they fall short of functioning as tears due to lack of nutrients and factors present in natural tears. Autologous serum drops being prepared from patient's body fluids are thus non-allergic and their biomechanical and biochemical properties are similar to normal tears. Their use in keratoconjunctivitis sicca was first described by Fox et al [1] in 1984 in their search for tear substitute free of potentially harmful preservatives. Later, Tsubota et al[2] reported that growth factors and vitamins present in serum eye drops have true epitheliotropic potential for ocular surface in sjogren's syndrome.

\section{Material and methods:}

Twenty six eyes of thirteen severe dry eye patients were enrolled in this study.

Inclusion criteria:

- Severe dry eye patients refractory to treatment with conventional artificial tears.

- Schirmer's test reading less than $5 \mathrm{~mm}$.

- Tear film breakup time less than or equal to 5 seconds.

- Presence of corneal and conjunctival staining with fluorescein.

- $\quad$ Grade IV symptoms

- No contraindications for blood extraction

Exclusion criteria:

- HIV

- $\mathrm{HCV}$

- $\mathrm{HBV}$

- Syphilis

Prior to starting of autologous serum on patients, following evaluations were performed-

* Subjective evaluation: Patients were asked to grade their symptoms on the format mentioned below. The grading was redone on each follow-up and benefit from treatment was assessed.

Grade O: No symptoms

Symptom grading

Grade I: Mild symptoms with no discomfort 
Grade II: Moderate symptoms with discomfort but no interference with daily activities

Grade III: Severe symptoms with discomfort but no interference with daily activities

Grade IV: Very severe symptoms with discomfort and interference with daily activities

* Objective evaluation: This was done by following tests-

Tear evaluation- Schirmer's test and tear film break up time.

Ocular surface evaluation- Fluorescein staining

Above mentioned tests were done at the start of treatment with autologous serum eye drops eight times per eye per day, at two weeks, at four weeks and six weeks.

III. Observations:

Table-1: Age distribution of patients

\begin{tabular}{|c|c|}
\hline Age in years & Number of patients \\
\hline $25-35$ & 3 \\
\hline $36-45$ & 4 \\
\hline $46-55$ & 4 \\
\hline $56-65$ & 2 \\
\hline
\end{tabular}

Table-2: Schirmer's test readings

\begin{tabular}{|c|c|c|c|}
\hline Schirmer's reading (mm) & $\begin{array}{c}\text { No. of eyes } \\
\text { At base line }\end{array}$ & At six weeks \\
\hline Less than 2 & 16 & 8 & 13 \\
\hline Less than 3 & 8 & 3 & 6 \\
\hline Less than 4 & 1 & 2 & 8 \\
\hline Less than 5 & 1 & 0 & 8 \\
\hline More than 5 & 0 & & 2 \\
\hline
\end{tabular}

Table-3: Tear film breakup time

\begin{tabular}{|c|c|c|c|}
\hline TBUT $(\mathrm{sec})$ & No. of eyes at baseline & No. of eyes at two weeks & No. of eyes at six weeks \\
\hline$<2$ & 14 & 6 & 2 \\
\hline$<4$ & 10 & 8 & 6 \\
\hline$<6$ & 2 & 8 & 7 \\
\hline$<8$ & 0 & 4 & 9 \\
\hline$<10$ & 0 & 0 & 2 \\
\hline
\end{tabular}

Table-4: Fluorescein staining

\begin{tabular}{|c|c|c|c|}
\hline Staining & No. of eyes at baseline & No. of eyes at two weeks & No. of eyes at six weeks \\
\hline Mild & 0 & 2 & 18 \\
\hline Moderate & 0 & 16 & 4 \\
\hline Severe & 26 & 8 & 4 \\
\hline
\end{tabular}

\section{Results:}

Twenty six eyes of thirteen patients belonging to age group of 25-65 years suffering from severe dry were evaluated. Of these, nine were female (69.23\%) and four were male (30.76\%). All patients had grittiness and foreign body sensation as presenting complaints, and were on some form of lubricants for treatment. Mean duration of their treatment with lubricants was $24.57+/-12.7$ months.

At baseline, Schirmer's test results were subnormal in all patients (mean $1.63 \mathrm{~mm}$ ), BUT was less than or equal to 5 seconds (mean 4.18). All patients had positive staining at baseline. On first follow-up at two weeks of treatment, mean Schirmer's score was $3.43 \mathrm{~mm}$ and value of mean BUT was found to be 6.62 seconds. There was substantial improvement in ocular surface disease as evidenced by mild or moderate staining in 18 eyes after treatment while 8 eyes continued to have severe staining. On last follow-up at six weeks, 22 eyes were symptom free, mean Schirmer's score recorded was $5.96 \mathrm{~mm}$, mean BUT was 9.08 seconds. Though all eyes showed evidence of ocular surface staining but there was shift noted in 22 eyes from severe to either mild or moderate degree. No complications were reported in this study.

\section{Discussion:}

This study confirms the beneficial effects of autologous serum in patients with severe dry eye. Although the number of patients enrolled in this study is not large, the improvements seen were clinically and statistically significant. Serum contains various factors that are also present in tears including vitamin A, epidermal growth factors, substance P, as well as proteins such as lactoferrins and lysozymes [3]. All these factors are essential for healthy functioning of ocular surface. Biochemical properties of normal human tears and serum are approximately similar with vitamin A, fibronectin, transforming growth factor beta been more in 
serum and surface immunoglobulin A more in tears [4]. Also, there is no difference between growth factor levels of autologous serum of dry eye syndrome and normal person [5]. Transforming growth factor beta is also believed to control epithelial proliferation and to maintain cells in undifferentiated state. Neural factors such as substance $\mathrm{P}$ are important for corneal epithelial migration. Human serum stimulates corneal fibroblast migration, proliferation and matrix metalloproteinase activity [6]. Presence of oil in serum may act as a replacement for lipid tear component produced by the meibomian glands. Impression cytology studies also suggest that autologous serum leads to upregulation of goblet cells and mucin expression in a dose dependent manner [7]. Autologous serum eyedrops improve conjunctival status too in dry eye syndrome patients [8]. Severe dry eye and ocular surface disease constitute a major management challenge. In this study, patients had significant improvement both subjectively and objectively after using autologous serum for six weeks.

\section{Conclusion:}

Autologous serum eyedrops were found effective and safe in treatment of severe dry eye disease, as evidenced by improvement in subjective assessment of symptoms, Schirmer's score, tear film break-up time and ocular surface staining.

\section{References:}

[1]. Fox RI, Chan R, Michelson JB, et al. Beneficial effect of artificial tears made with autologous serum in patients with keratoconjunctivitis sicca. Arthritis Rheum 1984; 27: 459-61.

[2]. Tsubota K, Goto E, Fujita H, et al. Treatment of dry eye by autologous serum application in Sjogren's syndrome. Br J Ophthalmol 1999; 83: 390-95.

[3]. Rocha EM, Pelegrino FS, de Paiva CS et al. GVHD dry eyes treated with autologous serum tears. Bone marrow transplant 2000; 25: 1101-03.

[4]. Geerling G, Honnicke K, Schroder C, et al. Quality of salivary tears following autologous submandibular gland transplantation for severe dry eye. Graefes Arch Clin Exp Ophthalmol 1999; 237: 546-53.

[5]. Bradley JC, Bradely RH, Mc Cartney DL, Mannis MJ. Serum growth factor analysis in dry eye syndrome. Clin Exp Ophthalmol 2008; 36(8): 717-20

[6]. Watson SL, Secker GA, Daniels JT. The effect of therapeutic human serum drops on corneal stromal stromal wound healing activity. Curr Eye Res 2008; 33(8): 641-52.

[7]. Kojima T, Ishida R, Dogru M, Goto E, Tsubota K. The effect of autologous serum eye drops in the treatment of severe disease; A prospective randomized case-control study. Am J Ophthalmol 2006; 136(2): 242-46.

[8]. Jirsova K, Hrdilckova E, Al Fakih A, et al. The application of the autologous serum eye drops results in significant improvement of the conjunctival status in patients with dry eye syndrome. Cesk Slov Oftalmol 2008; 64(2): 52-56. 